\title{
DES ACCENTS ÉTRANGERS AUX ABORDS DE L'UTOPIE BROSSARDIENNE
}

\author{
GHISLAINE BOULANGER
}

Université d'Ottawa

Résumé

Cet article analyse l'analogie entre une langue sexiste et une langue étrangère, et démontre comment cette comparaison met en jeu des dimensions identitaires qui seront progressivement refoulées aux frontières de l'utopie féministe imaginée par Nicole Brossard. Cette étude met en relief certains problèmes d'exclusion reliés à la disparition symbolique de l'accent, tels que l'effacement de l'identité juive dans le roman Picture theory.

Dans un chapitre préliminaire de son livre publié en I'an 2000, Narrative in the Feminine: Daphne Marlatt and Nicole Brossard, la critique Susan Knutson préconise un recours à des analogies entre divers modes d'oppression, tels le sexisme et le racisme, même si, concède-t-elle, ces comparaisons risquent, par exemple, de détourner notre attention des problèmes de racisme en nous incitant plutôt à focaliser sur des formes de sexisme dont seraient victimes des femmes blanches $(29)^{1}$. Consciente de ces dangers, elle adopte pour sa part une approche intersectionnelle qui lui permet d'esquiver ces pièges dans le cadre de ses propres théories sur le « générique (masculin) »(29). Cependant, malgré I'importance qu'elle accorde à ces stratégies argumentatives, Knutson ne signale pas la présence d'analogies semblables dans les principaux textes brossardiens qu'elle étudie, soit le roman Picture theory, paru en 1982, et une conférence connexe prononcée la même année, « De radical à intégrales ». Or, si cette théoricienne réussit à contourner les problèmes d'exclusion pouvant découler de tels procédés, cela ne signifie pas que Nicole Brossard y parvient également. En effet, une analyse approfondie de I'analogie de la langue étrangère sur laquelle s'appuie la théorie-fiction brossardienne démontrera comment cette comparaison met en jeu des dimensions identitaires qui seront progressivement refoulées aux frontières de l'utopie féministe.

Afin que la scène amoureuse dépeinte dans Picture theory atteigne des proportions utopiques, afin qu'advienne l'image tridimensionnelle de la femme « intégrale » (PT 166) ${ }^{2}$, l'une des deux amantes, le personnage de Claire Dérive Stein, devra ainsi perdre son accent linguistique ( $P T$ 160). Cette métamorphose graduelle conduisant à l'émergence de métaphores utopiques a pour fondement une analogie par laquelle Brossard souhaite élucider les liens langagiers unissant les femmes au «système patriarcal » $(D R \text { 88 })^{3}$ : le rapport des femmes à une langue maternelle sexiste ressemblerait à celui de certaines «personnes » à l'égard d'une langue étrangère. Faussement maternelle, la langue dite patriarcale serait aussi étrangère aux femmes qu'une langue seconde, et cette distance s'exprimerait par l'entremise d'un accent. Mais lorsque Brossard compare la situation des femmes à celle des «personnes qui ont un accent » (DR 92), prend-elle en considération, par exemple, la réalité vécue par 
des femmes migrantes aux prises avec la xénophobie d'une culture d'accueil? Une théorie qui prône la disparition symbolique d'un accent linguistique ne risque-t-elle pas de reproduire un type d'ethnocentrisme semblable à celui que doivent affronter les femmes dont l'accent est littéralement méprisé? Autrement dit, est-il réellement possible de recourir à l'analogie de la langue étrangère uniquement au sens figuré, sans que n'interfèrent certaines nuances sémantiques inhérentes au sens littéral? En effet, comment se fait-il qu'une seule des deux amantes doive, pour accéder au statut de «femme intégrale», se départir explicitement de son accent? La suppression de l'accent recouvre-t-elle d'autres disparitions? Pour mieux saisir les implications théoriques et idéologiques de l'analogie de la langue étrangère déployée dans le roman Picture theory, il convient, en premier lieu, de dégager les prémisses et les incidences de cette comparaison dans l'essai «De radical à intégrales », où Brossard en a fait la pierre angulaire de son argumentation.

\section{1. « De radical à intégrales 》}

Commençons ainsi par examiner plus attentivement les prémisses de l'analogie qui nous intéresse, soit le rapport entre certains sujets et une langue dite étrangère. D'une part, Brossard se réfère à des «personnes qui », ayant assimilé la langue étrangère à divers degrés, s'expriment avec « un accent » (DR 92); et, d'autre part, les sèmes linguistiques et spatiaux se fusionnent en l'image «[ $\left.d^{\prime}\right]$ une terre sémantique étrangère » (DR 89). Les notions d'accent étranger et de terre étrangère présupposent des foyers perceptifs depuis lesquels seront distinguées les dimensions étrangères ; des perspectives qui, d'abord au niveau du phore (ou du comparant), puis au niveau du thème (ou du comparé), s'opposeront les unes aux autres de manière à sous-tendre éventuellement un tri des différences entre femmes.

Au niveau du phore, les « personnes qui ont un accent » se trouveraient ainsi sur un territoire qui leur est « étranger »(DR 92), comme lorsque le personnage de Claire Dérive Stein, originaire des États-Unis, séjourne à Montréal. Puisque les signes de l'inconnu sont déterminés comme tels à partir d'un centre formant la sphère du connu, un espace pourrait être qualifié d'étranger par des sujets profondément attachés à leurs origines géographiques et/ou ethno-culturelles, lesquelles conserveront toute leur valeur de noyau primitif et essentiel. La présence de ces «personnes» en milieu « étranger» s'expliquerait par exemple en termes d'émigration, de voyage ou d'exil. Sous cette première optique, ce ne sont pas ces individus qui portent les marques du différent ou de l'étranger, mais bien la culture d'accueil. Ces rôles peuvent toutefois s'inverser lorsque s'impose un autre angle de vision. Les signes de l'inconnu pourraient être considérés comme tels par le groupe de référence dominant du territoire d'accueil. Depuis un foyer perceptif idéologiquement inaccentué, ou apparemment non marqué, ce groupe dominant pourrait ainsi dire des personnes venues d'ailleurs qu'elles s'expriment avec un accent. Il s'agirait non 
seulement de percevoir une langue étrangère, mais aussi de discerner des éléments étrangers dans la langue majoritaire. En l'occurrence, le rapport à la terre d'accueil pourrait se sceller d'ostracisme, l'accent étranger constituant l'indice d'une différence réprimée ou dépréciée par le groupe dominant, comme cela peut se produire dans certaines circonstances décrites par I'historienne Lise Noël:

Aux oreilles du dominant, l'accent et l'intonation sont donc révélateurs de l'appartenance à un groupe inférieur. Sa propre intonation lui paraissant normale, il ne la remarquera pas, pas plus qu'il ne considérera qu'il a lui-même un accent comparé aux autres. Qu'il soit réel ou qu'on le lui prête, le ton adopté par le dominé quand il parle donnera aussi la mesure de sa compétence. (50)

Or, s'il importe de faire ressortir ces univers confrontés les uns aux autres au niveau du phore, c'est qu'un phénomène semblable se produira au niveau du thème, lorsqu'un centre essentiellement féminin s'opposera à un centre patriarcal.

Comme on le sait, c'est à titre de relation aliénante que le rapport à la langue étrangère se compare à celui qui relie le sujet féminin à une langue patriarcale: «D’avoir fait en sorte, par la force du code et de la loi et par habitude ensuite, que chaque femme fasse sienne la substance sémantique patriarcale est la plus grande réussite du patriarcat »(DR 90). Parce qu'elle rature le féminin, subordonne celui-ci ou l'assimile au masculin, la langue maternelle est donc qualifiée d'étrangère. Étant donné que les signes de l'étranger sont déterminés à partir d'un foyer perceptif qui s'en distingue, la théorie brossardienne présuppose un espace féminin originel d’où émanerait une résistance, consciente ou non, aux codes dominants. L'accent se développe en dépit de l'assimilation à la culture androcentrique, tel qu'en fait preuve l'énoncé suivant: «Mais cela n'a pourtant pas empêché que bien assimilée, mal assimilée ou non assimilée, cette langue étrangère nous la parlons toutes avecun accent »(DR 90). Esquivant l'emprise patriarcale qui ne peut en freiner la manifestation, l'accent signale avant tout « un écart par rapport à la norme » (DR 90), un écart que l'auteure investit de pouvoirs subversifs. L'accent consiste ainsi en «une augmentation d'intensité à l'emploi de certains mots, sur certains sons »(DR 90), qui occasionnerait des déformations sémantiques substantielles, car «ce qui est intense a une force par laquelle nous dépassons la mesure ordinaire, la norme » (DR 91).

Le dépassement envisagé par l'écrivaine québécoise n'a toutefois de véritable portée politique que si «la pratique collective de l'écart sémantique » (DR 90) est « suivie » d'une «fréquentation des femmes » entre elles (DR 91). Quoique collectif, l'exercice «de l'écart sémantique » ne provient pas initialement d'une différence commune, ou d'une perspective commune à toutes les femmes, mais bien plutôt de différences variées, de perspectives dont la diversité s'avère propice à la dérogation: «placées d'où nous sommes, multiples et différentes, nous faisons un usage ambigu de plusieurs mots et cet usage ambigu né de notre ambivalence à l'égard du sens fait que collectivement, nous nous trouvons momentanément à déroger au sens habituel des 
mots » (DR 96). Oscillant vers I'inusité, un « déferlement de mots polyvalents et multi-directionnels » provoquerait enfin «l'éclat du sens unique » patriarcal (DR 96), « [brisant] I'homme comme universel » (DR 96). Cependant, à « cette étape de l'éclat du sens » (DR 96), le discours brossardien procède à certains revirements.

Tout d'abord, la primauté d'un « sens issu de nous» (DR 98) s'affirme non seulement à l'encontre du «sens unique patriarcal», mais aussi à l'encontre de «l'effet de polyvalence» (DR 96) suscité par les « différences entre [femmes]» (DR 92). Malgré leur propriété subversive, les sonorités accentuées peuvent sembler inintelligibles, et, pour cette raison, occasionner une mésentente: « intenses, diverses et rassemblées, des femmes échangent leurs vues au moyen de mots étrangers à leurs perceptions, à leur vécu, et en cela même éprouvent de la difficulté à s'entendre sur le sens à donner aux mots et par voie de conséquence à leur vie, à leurs projets » (DR 95), car, selon l'auteure, « ce qui caractérise les personnes qui ont un accent, c'est qu'elles déforment les sons et que par conséquent elles risquent chaque fois qu'elles s'expriment en langue étrangère de créer des malentendus, des équivoques, voire même du non-sens » (DR 92). Associant les différences à des différends, Brossard tente de freiner le flux du divers et du polysémique, «pour que la vie ait un sens, pour que la vie soit accessible dans sa lisibilité, pour qu'elle ne soit pas continuellement effet de contradiction. Pour mettre un terme à l'ambiguïté » (Cotnoir 191). Elle croit donc nécessaire d'uniformiser les signifiés du mot «femme »: une culture au féminin, insiste-t-elle, ne pourrait advenir qu'à la condition de « faire sens collectivement » (DR 97): « Il faut une base minimale d'entente sur le sens à donner aux mots pour que nous puissions songer à l'émergence $d^{\prime}$ une culture qui nous ressemble $\gg^{4}$ (DR 98). Interdisant une certaine plurivocité, le «faire sens » collectif se soutient de ressemblances qui présupposent une entente implicite sur « ce que nous sommes »: «Il faut donc [...] faire en sorte de fréquenter des êtres qui nous ressemblent suffisamment pour créer un lien (affinité, rapport, liaison, analogie), ce qui permet de donner suite à ce que je suis, à ce que nous sommes » (LA 118), déclare ainsi l'auteure dans une autre conférence donnée la même année que fut publié Picture theory.

Sur cet accord tacite anticipé, Brossard fonde un modèle qu'elle souhaite unificateur, proposant à ses lectrices une image identitaire où la pluralité s'appréhende pourtant par le biais d'un signe unique: «L'Intégrales est radicale. [...] elle est 'symbola' pour toutes, un signe de reconnaissance. Figure, image, métaphore, elle fait toujours sens et corps avec le sens qu'elle donne aux mots » (DR 100). Malgré la marque du pluriel accolée à cette figure, celle-ci fonctionne syntaxiquement au singulier; d'ailleurs, lorsque «cette femme intégrale que nous sommes, cette idée de nous » (DR 87) a surgi pour la première fois, dans Picture Theory, son nombre grammatical se réduisait alors à l'unicité: «l'utopie l'intégrale » (PT 166). Ce problème est d'ailleurs bien cerné par la théoricienne Diana Fuss:

It is common practice in social constructionist argumentation to shift from the singular to the 
plural in order to privilege heterogeneity and to highlight important cultural and social differences. Thus, woman becomes women, history becomes histories, feminism becomes feminisms, and so on. While this maneuver does mark a break with unitary conceptual categories (eternal woman, totalizing history, monolithic feminism), the hasty attempts to pluralize do not operate as sufficient defenses or safeguards against essentialism. The plural category «women », for instance, though conceptually signaling heterogeneity nonetheless semantically marks a collectivity; constructed or not, « women » still occupy the space of a linguistic unity. [...] The essentialism at stake is not countered so much as displaced. (4)

Ainsi, « [I'] effet de polyvalence » qui fit initialement éclater le «sens unique patriarcal », se voit à présent freiné pour permettre le tracé d'un groupe de référence essentiel. Comme l'explique Annamarie Jagose, «the very fixity of meaning Brossard attributes to patriarchal language is more properly attributed to her own articulation of the category 'women' and her reification of that category as the origin of an alternative, feminine reading, a 'sense which originates with us' (Aerial Letter 135) 》(66).

Afin de mieux fixer les contours «d'une culture qui nous ressemble ( DR 98), l'écrivaine québécoise procède à un tri des différences entre femmes. C'est ainsi que s'opère, à « [I']étape de l'éclat du sens 》 (DR 96), un subtil glissement sémantique concernant «nos différences », un renversement axiologique dont témoigne le paragraphe suivant:

Alors que dans un premier temps, I'activation de nos différences confrontées au sens unique patriarcal a permis l'éclat du sens et sa vacance, il faut entrevoir désormais nos différences comme provenant d'un premier sens issu de nous. En d'autres termes, les différences issues du sens patriarcal ont permis un mouvement, alors que les différences issues de nous comme centre originel permettront l'émergence d'une culture au féminin. (DR 98)

Malgré une apparente continuité entre les deux «temps » décrits ci-dessus, la seconde phase de ce cheminement, au lieu de s'harmoniser avec la première, se retourne plutôt contre celle-ci de manière contradictoire. « Dans un premier temps », Brossard postule l'existence de «différences confrontées au sens unique patriarcal », c'est-àdire, selon les acceptions du participe «confrontées », des différences qui font face au patriarcat ou sont mises en présence de celui-ci. Cet affrontement présuppose une distinction préalable entre « nos différences » et le « sens unique patriarcal», une distance qui renvoie à cet «écart» que creuse l'accent «par rapport à la norme » patriarcale (DR 90). Mais dans un deuxième temps implicite, l'auteure se ravise et désavoue cette croyance initiale lorsqu'elle tente de modifier notre perception des différences (« il faut entrevoir désormais nos différences »), niant que ces dimensions identitaires aient pu émaner, «dans un premier temps », d'un « premier sens issu de nous ». Ce nouvel impératif («il faut ») entraîne une altération des «termes », une autre version: même si les dissemblances différaient antérieurement d'une norme patriarcale qui leur était étrangère et avec laquelle elles entraient en confrontation, ces différences devront «désormais » être perçues comme étant «issues du sens patriarcal ».

Si soudain puisse-t-il sembler, ce revirement était pourtant annoncé dans l'incipit du texte, où l'auteure 
décrivait « un système de valeurs qui, d'une part, détermine ce qui fait sens ou non-sens et qui, d'autre part, normalise le sens de manière à ce qu'excentricité, marginalité et transgression puissent être identifiées comme telles afin d'être contrôlées si besoin est » (DR 88). Or, dans un contexte où les différences initiales font l'objet d'une seconde interprétation, il s'ensuit logiquement que l'accent, qui symbolise ces différences, acquiert lui aussi une nouvelle signification. Comme nous le découvrirons davantage à la lecture de Picture theory, l'accent deviendra, dans un deuxième temps, tout aussi étranger au « centre originel » que ne l'était la langue étrangère. Bien que l'accent représente, «dans un premier temps», un « écart par rapport à la norme » patriarcale, il se transforme ensuite, selon la deuxième perspective prônée par Brossard, en un signe d'assimilation au patriarcat: l'accent devient le signe d'une différence déterminée comme telle par le centre patriarcal (« les différences issues du sens patriarcal »), un signe indirectement, voire intrinsèquement, patriarcal. L'accent jouera donc deux rôles successifs, suivant les phases antinomiques de la théorie brossardienne : après avoir signifié « un écart par rapport à la norme » patriarcale, l'accent signifiera un écart par rapport à une nouvelle norme féministe, ou, plus précisément, par rapport à l'utopie brossardienne. L'accent étranger (ou les différences qu'il symbolise) n'est pas uniquement désigné comme tel à partir d'un centre patriarcal qui «détermine ce qui fait sens ou non-sens »; l'accent est également défini comme tel à partir d'un «centre originel », d'une « culture au féminin » d'où seront écartées les femmes qui s'exprimeraient avec l'accent étranger d'une culture patriarcale à laquelle elles seront associées.

En effet, le découpage en deux «temps» structurant le passage du patriarcat à une «culture au féminin » consiste en un renversement de situation qui scinde la catégorie «femmes » en deux groupes opposés : I'un, essentiellement féministe radical, et l'autre, essentiellement patriarcal (DR 97). Bien que l'émancipation anticipée par l'écrivaine dépende du potentiel subversif des différences qui, « dans un premier temps», provenaient d'un foyer de résistance face au patriarcat, dans un deuxième temps, elle dénie cet apport initial de la diversité en réduisant son origine et sa portée au «sens unique patriarcal » (DR 95). Se retournant contre la première phase, la seconde se fonde non plus sur l'idée de continuité, mais sur celle de discontinuité, de rupture. Tout se produit comme si Brossard détruisait la passerelle qui lui avait pourtant permis de franchir le fossé entre la « terre sémantique étrangère » $(\mathrm{DR} 89)$ et le «territoire imaginaire [...] féministe » (DR 98). Citant Marilyn Ferguson à l'appui, l'auteure de La Lettre aérienne croit que « le problème, c'est qu'on ne peut saisir le nouveau paradigme tant qu'on n'a pas abandonné I'ancien » (LA 81). Or, s'il ne s'agissait que d'un simple changement de paradigmes, ce revirement serait sans doute moins problématique. Ce qui pose problème, c'est qu'à chacun de ces paradigmes sont assignés des groupes de femmes ou des dimensions identitaires spécifiques, et que I'essentialisation de figures utopiques a pour corollaire l'essentialisation de figures patriarcales. Ainsi, I'agentivité 
potentiellement subversive des femmes qui se distinguent des féministes radicales se voit-elle soudainement minorée de manière à ce que puisse être instaurée une antithèse définitive entre un « centre patriarcal » et un « centre originel». Une fois différentes perspectives assimilées au «sens unique patriarcal», une fois leur virtualité subversive remise en question, Brossard fusionne le féminin au patriarcat en l'image statique de la «femme patriarcale»: «C'est ici que moi je te dis adieu femme patriarcale» (DR 97). Or, qui sont-elles exactement ces «femmes fragmentées» (DR 97), délaissées aux limites de l'utopie brossardienne? En quoi consistent-ils, ces «fragments » identitaires dont doivent se détourner celles qui aspirent à devenir « intégrales »? Le « système de valeurs féminines » (LA 74), auquel l'auteure invite «les femmes synchrones » (LA 74, PT 189) à adhérer, pourra-t-il, dans Picture theory, esquiver les pièges « [d']un système de valeurs qui [...] détermine ce qui fait sens ou non-sens » (DR 88)?

\section{Picture theory}

Dans le roman brossardien, sens littéral et sens figuré s'entremêlent au sein d'un même personnage: au sens littéral (phore ou comparant), Claire Dérive Stein s'exprime en français avec l'accent anglais de sa langue maternelle; au sens figuré (thème ou comparé), son accent symbolise également un rapport à la langue patriarcale. S'il est possible d'affirmer que l'accent de Claire s'entend littéralement au sens ethnique, c'est que son accent est perçu et déterminé comme tel par la narratrice, son amante, qui perçoit aussi d'autres accents étrangers, comme lorsqu'elle décrit « certains [hommes qui] avaient un accent » (PT 99). Bien que I'analogie de la langue étrangère confère un second sens à l'accent de Claire, celui-ci fonctionne à la base sur le même plan sémantique que l'accent de certains personnages masculins. La narratrice, nommée Michèle Vallée ou M.V., perçoit d'ailleurs les origines nationales de Claire de façon stéréotypée, réduisant parfois son américanité à des clichés culturels essentialistes : «Claire revenait avec le vin, hors d'elle, parlait bitch, dyke, sentait I'américaine à plein nez, ultra modern style new-yorkais » (PT 95). Nous découvrirons ainsi comment ce sens littéral sous-tendra le passage ultérieur à un sens figuré, et comment ce passage s'inscrira dans le cadre d'un dualisme entre des « différences » identitaires «issues du sens patriarcal » et des « différences issues de nous comme centre originel 》(PT 98).

Comme on a pu le voir brièvement, la métamorphose de Claire en femme « intégrale » est symbolisée par la perte de son accent. Bien que cette transformation s'accomplisse par l'entremise d'une scène amoureuse entre Claire et la narratrice, et quoique, selon l'auteure, cette scène «[fasse] que chacune des deux femmes devient intégrale » (Cotnoir 195), en réalité, seule l'une des deux amantes possède un accent explicitement voué à la disparition. En effet, même si l'essai «De radical à intégrales » spécifie que toutes les femmes s'exprimeraient 
avec un accent (LA 90), le roman ne contient aucune précision indiquant que la voix de la narratrice serait doublement accentuée comme l'est la voix de Claire, c'est-à-dire à la fois au niveau littéral et au niveau figuré. La narratrice met principalement l'accent sur l'accent de Claire, comme I'ont fait une majorité de critiques et l'auteure elle-même ${ }^{5}$, parce que cet accent, si peu marqué soit-il, est marquant au point de devenir l'objet d'une obsession: « Le répondeur: Je suis Claire Dérive. La voix était belle, presque sans accent. [...] J'étais obsédée par la voix de Claire Dérive [...]. Elle n'avait dit que son nom. [...] La vie mode d'emploi, presque sans accent » (PT 42). Tel que souligné par Michèle, il suffit à Claire de prendre la parole pour que son accent soit perçu comme tel : nul besoin de procédés stylistiques pour que cette voix se distingue. Il suffit à la narratrice de rapporter l'existence d'un accent pour le faire exister, et, plus tard, de relater sa disparition pour le faire disparaître.

Bien que les accents signalés explicitement dans Picture theory se différencient des autres voix indépendamment de toute stratégie stylistique, la critique Dawn Thompson coit pour sa part que la voix narrante serait implicitement accentuée au féminin sur le plan symbolique (16-42). Malgré ses mérites, son analyse ignore toutefois les deux versants de l'analogie de la langue étrangère, et, conséquemment, ne mentionne ni le double sens de l'accent de Claire, ni l'accent des personnages masculins que perçoit la narratrice. Cette théoricienne ne prend pas en considération la composante ethnique de l'accent, un aspect qui permet à Brossard de lever la barrière entre les genres pour réunir femmes et hommes sous une même catégorie, celle des «personnes qui ont un accent » (DR 92), et pour déplacer certaines «différences » culturelles sous l'égide du patriarcat, comme en témoigne l'image de la «femme patriarcale ». De fait, sur le plan diégétique, la voix narrante demeure inaccentuée à I'oreille des autres personnages, car c'est la narratrice qui occupe une position centrale, un foyer perceptif depuis lequel sont déterminés comme tels les accents d'autrui. Lorsque Claire s'exprime en français, elle n'a littéralement d'accent que parce que sa langue maternelle est l'anglais, et que cet accent est considéré comme tel par son amante dont la langue maternelle est le français. Pour reprendre les explications de Lise Noël, nous pourrions ainsi dire que «sa propre intonation lui paraissant normale, [Michèle] ne la remarquera pas, pas plus qu'[elle] ne considérera qu'[elle] a [elle]-même un accent comparé aux autres » (50). En effet, si l'intonation de la voix narrante passe inaperçue, ou si la narratrice n'a aucun accent à perdre, c'est que sa position ethno-culturelle fonctionne d'emblée comme une norme à partir de laquelle sont déterminés certains signes différentiels. Passant inaperçue, cette position ethno-culturelle influence subtilement la détermination de nouvelles normes féministes en fonction desquelles certaines dimensions identitaires seront redéfinies comme étant d'origine patriarcale.

Allant de soi, la voix narrante donne donc à entendre d'autres accents, et, plus particulièrement, celui de Claire, appelé à disparaître. Cette disparition se jouera à deux niveaux, puisque les nuances ethniques inhérentes au sens littéral cohabitent avec le sens figuré et contribuent à le façonner. Autrement dit, la suppression d'un 
accent dont le sens est double recouvre logiquement une double disparition, entraînant l'effacement de dimensions ethniques qui, comme nous le verrons, seront progressivement assimilées au patriarcat. Ainsi, l'élimination de l'accent impliquera une double métamorphose : la transformation de Claire en femme « intégrale » découlera d'une modification préalable de son rapport à la langue/mère. Afin que son accent représente non seulement une difficulté avec le français langue seconde, mais aussi une difficulté avec l'anglais comme langue faussement maternelle, il faudra que le rapport à la langue maternelle se transforme en un rapport à la langue patriarcale. Cette métamorphose s'accomplira par l'intermédiaire d'une remise en question du personnage de la mère, Sarah Dérive Stein, et, corollairement, de la relation mère-fille.

Le personnage de Sarah incarne initialement un faisceau de différences identitaires propices à I'autodétermination: «Leur mère autrichienne et communiste disait que juive, elle se sentait femme et intellectuelle » (PT 23). Non seulement enracine-t-elle sa féminité dans un espace ethno-culturel spécifique, celui de la judéité, mais cet espace abrite, outre des origines européennes, une forme de résistance à l'ordre établi, par le biais du communisme. S'entrecroisent, sur le territoire new-yorkais où vit cette femme, les positionnements divers et subversifs dont Brossard reconnaissait initialement la valeur dans son essai « De radical à intégrales ». Cependant, au décès de ce personnage s'ensuit une redéfinition de son identité, comme si le fait que Sarah ait ellemême défini sa propre identité du temps de son vivant s'avérait désormais insuffisant ou sans validité. Ainsi, Claire cherchera-t-elle ailleurs les indices de l'identité de sa mère: «It is obvious that she was a woman, isn't it, Michèle? » (PT 136); «Yes, she was a woman, wasn't she? » (PT 138); « I need proofs that she was a woman. I need to know » (PT 139). En réponse à ces interrogations, l'identité féminine de Sarah sera reconstruite de telle sorte que nous pourrons observer un autre revirement.

La critique Lianne Moyes a décelé d'importants liens intertextuels entre I'oeuvre de Gertrude Stein et le roman Picture theory, allant jusqu'à « suggérer » l'idée d'une relation étroite entre cette écrivaine et le personnage de Sarah Dérive Stein (217). Selon Moyes, la déconstruction de l'identité de Sarah serait typiquement steinienne, puisque Gertrude Stein s'employait à déconstruire la notion d'identité et les catégories de genres (gender). Comme l'explique Moyes, «the issues raised by this character [...] are issues Picture Theory raises about its own production » (217). Cependant, ces questions ne sont pas soulevées par le personnage («by this character»), mais bien plutôt à propos de ce personnage (about «this character»). Certes, la remise en cause de l'identité féminine de Sarah peut être attribuée à la pensée de Gertrude Stein, mais ce n'est pas le personnage de la mère qui désavoue sa propre identité; ce sont les personnages de Claire et de Michèle qui procèdent à sa réévaluation. Moyes s'en tient aux descriptions extérieures à ce personnage, et passe sous silence le discours de Sarah sur sa propre identité, lorsque celle-ci fonde sa féminité sur sa judéité. Alors que l'identité juive permet à Sarah de se 
sentir «femme et intellectuelle»(PT 23), Gertrude Stein, comme le précise Moyes, « [refused] conventional sex/gender categories and specifically [refused] to identify herself as a 'woman writer' (217). Il importe donc d'établir une distinction entre l'identité revendiquée par le personnage de la mère et la remise en question de cette identité par Claire et Michèle, suite à son décès.

Ainsi, la narratrice commencera-t-elle par pousser les manifestations de la diversité jusqu'à l'éparpillement; ce qui, semble-t-il, minerait tout effort d'émancipation : «Sarah, cette mère abstraite, cette femme studieuse [...]. Docte et tourmentée, tournant page après page une vie sans résumé, toujours exposée à la dispersion à travers les courants idéologiques de son temps » (PT 137). Lianne Moyes associe cette dispersion à Gertrude Stein et au phénomène de «loosing one's identity in the process of writing » (217), mais, si I'on tient compte de l'identité juive de la mère, qui émigra de l'Autriche vers les États-Unis, comment ne pas songer à la dispersion bien connue des personnes juives à travers le monde, à la diaspora? Comme dans l'essai « De radical à intégrales », pluralité et résistance convergeront finalement vers le «sens unique patriarcal », alors que Claire cherchera vainement dans les livres les traces de sa mère: «Là-dessus, les livres ne disent rien/rien au sujet des femmes qui le sont devenues, occupées toute une vie à défendre de tout leur être les droits de I'Homme » (PT 139). Certes, la narratrice concède que «Sarah Dérive Stein fut une femme » (PT 140), mais elle ne mentionne pas sa judéité, et c'est avec cette omission que coïncide la disparition subséquente du nom juif de Claire (PT 141), comme nous le verrons davantage un peu plus loin. Pour l'instant, nous assistons à la redéfinition progressive de l'identité de la mère, laquelle finit par correspondre à l'image de la «femme patriarcale », si l'on se fie à la définition qu'en propose Brossard dans La lettre aérienne: « une femme patriarcale, c'est-à-dire une femme qui épouse le sens que I'Homme donne à la vie, donc à la sienne englobant la femme » (LA 115). Ainsi, Sarah « [s'entourait] de jeunes poètes (au masc.) » (PT 118) et « jouissait de la vie dans les meetings et les conférences qui défendent les droits de I'Homme et la liberté de parole »(PT 83). Bien que Sarah ait enraciné son identité féminine dans la judéité, la narratrice range plutôt ce personnage parmi les «femmes qui le sont devenues [en défendant] les droits de I'Homme » (PT 139), ce qui permet de déplacer la judéité sous l'égide du patriarcat et de reconstituer cette femme juive en «femme patriarcale». Le lien entre les traditions juives et le patriarcat est d'ailleurs accentué par une référence à « [I']entrée [du fils] dans le monde des hommes, jour de Bar Mitzva » (PT 83). Brossard semble ainsi vouloir contester, par l'entremise du personnage de Sarah, les expressions « une femme est un homme » (DR 93) et « une femme c'est moi » (DR 94). Le premier énoncé « fonderait le sentiment que nous avons le droit et même le devoir de revendiquer au nom de l'homme: justice, liberté, fraternité, égalité » (DR 93), tandis que le second, malgré ses possibilités «polysémique[s] (parce que le moi prononcé par chaque femme a un sens différent) »(DR 93), ne serait qu'une « autre définition de soi-femme qui sous-entend que c'est 
aussi au nom de I'humanité (c'est-à-dire de I'homme qui sommeille en nous) que nous pouvons revendiquer autonomie, subjectivité, individualisme et créativité »(DR 94). Le rayonnement de différences et de résistances émanant du personnage de Sarah se condense donc en l'image de la femme/mère patriarcale, une figure identitaire dont se détourne Brossard : «L'origine n'est pas la mère, mais le sens que je donne aux mots et à I'origine, je suis une femme » (DR 97). L'auteure de Picture theory « [voulait] à tout prix éviter de répéter que la première femme est une mère » (Cotnoir 180).

Ce détournement d'origine est illustré par le rapport de Claire Dérive Stein à ses propres racines culturelles, comme en témoignent non seulement son accent, mais aussi son nom. Lorsque Claire s'exprimait en langue seconde, son accent préservait l'empreinte de sa langue maternelle, d'une langue symbolisant I’héritage maternel, un héritage juif, diversifié et rebelle à certains discours dominants. Ce n'est toutefois qu'après le décès de Sarah, lorsque la fille tentera de reconstituer l'identité féminine de sa mère, que Claire Dérive Stein se « [métamorphosera] en femme studieuse » comme le fut Sarah, et qu'elle portera son nom juif (PT 139). À travers cette quête d'identité, la judéité ne sera jamais mentionnée, mais elle sera représentée par cette mutation onomastique. Cependant, une fois I'identité maternelle reconstruite en «femme patriarcale », le nom Claire Dérive sera à nouveau dissocié du nom Stein, et apparaîtra sans ce dernier (PT 141). Selon Michèle Vallée, «Dérive était un nom qu'il fallait savoir mériter en dehors des questions de famille » (PT 95), comme si le nom Stein, pourtant relié à I'Histoire du peuple juif, ne méritait pas autant de déférence. Plus tard, au terme d'une dernière section consacrée à la mémoire de Sarah, la parole de Claire Dérive s'énoncera enfin « sans accent » (PT 160). Avec la perte de l'accent disparaît ainsi une dimension ethnique, une disparition consolidée par l'effacement corollaire du nom juif. Parce que l'accent et le nom Stein symbolisent un lien étroit avec une femme/mère juive redéfinie en femme/mère patriarcale, Claire doit s'en départir afin d'accéder à « l'utopie l'intégrale » (PT 166). La narratrice de Picture theory peut ainsi repousser dans la zone patriarcale certaines dimensions identitaires qui lui étaient étrangères: sans accent ni nom juif, seules subsistent des «femmes synchrones » (PT 189), des amantes « accouplées compatibles » (PT 192), car, tel que précisé en annotation, «in order for the one to represent the form of the other, there must be something that is the same in both » (PT 192). Entre les amantes se tissent des liens analogiques, puisque selon l'auteure, rappelons-le, « il faut donc, si l'on veut poursuivre la plus juste image de soi et l'exposer, faire en sorte de fréquenter des êtres qui nous ressemblent suffisamment pour créer un lien (affinité, rapport, liaison, analogie), ce qui permet de donner suite à ce que je suis, à ce que nous sommes » (LA 118).

Aux frontières de l'utopie féministe, il est finalement possible d'imaginer, parmi les «défroques de femmes fragmentées » (DR 97), dispersées, le souvenir d'une femme juive redéfinie en femme patriarcale, d'une 
mère juive dont I'héritage culturel, onomastique et linguistique disparaît progressivement au profit «d'une culture qui nous ressemble » (DR 98), comme si plusieurs d'entre «nous » n'étaient pas juives et fières de l'être. Avec I'élimination symbolique de l'accent s'effacent ainsi, au niveau littéral, d'autres dimensions identitaires, comme en témoigne le rejet du nom de Stein. Il est vrai que le féminisme brossardien s'ouvre, « dans un premier temps », aux nombreuses « différences » entre femmes; mais ces « différences » sont par la suite départagées selon un dualisme fondamental entre féminisme et patriarcat, et l'agentivité des femmes qui se distinguent des féministes radicales, ou que l'écrivaine considère comme des «femmes patriarcales», se voit soudainement dévalorisée, comme si leurs « différences » mettaient en péril l'idéal d'une «culture au féminin ». Une vingtaine d'années après la publication de Picture theory, Nicole Brossard parvenait à la conclusion suivante :

This said, I think that we, a necessary, loving we of solidarity that figures large in certain of my books, is probably what has taken me furthest. A vital we linking me time and again with my sister, my kind, a we releasing us from those thousands of slipknots tied with patriarchal lies. A we weaving that luminous wisp of utopia deep in our eyes. Someone said to me one day, « Don't you think there are women whom your we doesn't suit ? » Probably. So what? (Giant Nature 154)

http://www.brocku.ca/cfra/voixplurielles05-02/index.html 


\section{Bibliographie}

Altman, Meryl. "How Not To Do Things with Metaphors We Live by." College English 52.5 (1990): 495-506.

Brossard, Nicole. Picture theory. Montréal: Nouvelle Optique, 1982.

. «De radical à intégrales. » La Lette aérienne. Montréal: Remue-Ménage, 1985. 87-103.

. La Lette aérienne. Montréal: Remue-Ménage, 1985.

. The Aerial Letter. Trad. Marlene Wildeman. Toronto: Women's Press, 1988.

. Langues obscures. Montréal: L'Hexagone, 1992.

. "The Giant Nature of Words and Silence around Identity." Trans. Patricia Claxton. Fluid Arguments. Toronto : The Mercury Press, 2005. 146-161.

Cotnoir, Louise, Lise Guèvremont, Claude Beausoleil et Hugues Corriveau. «Entretien avec Nicole Brossard sur Picture theory. » La Nouvelle barre du jour 118-119 (1982): 177-201.

Fuss, Diana. Essentially Speaking: Feminism, Nature \& Difference. New York et London: Routledge, 1989.

Grillo, Trina et Stephanie M. Wildman. "Obscuring the Importance of Race: The Implication of Making Comparisons Between Racism and Sexism (or Other -isms)." Critical White Studies: Looking Behind the Mirror. Dir. Richard Delgado et Jean Stefancic. Philadelphie: Temple University Press, 1997. 619-626.

Knutson, Susan. Narrative in the Feminine: Daphne Marlatt and Nicole Brossard. Waterloo: Wilfrid Laurier University Press, 2000.

Jagose, Annamarie. Lesbian Utopics. New York et London: Routledge, 1994.

Noël, Lise. L'Intolérance - Une problématique générale. [Montréal/Paris]: Boréal/Seuil, Boréal Compact, 1991 [1989].

Moyes, Lianne. "Composing in the Scent of Wood and Roses: Nicole Brossard's Intertextual Encounters with Djuna Barnes and Gertrude Stein." English Studies in Canada 21.2 (1995):206-225.

Spelman, Elizabeth V. Inessential Woman: Problems of Exclusion in Feminist Thought. Boston: Beacon Press, 1989.

"Changing the Subject: On Making Your Suffering Mine." Fruits of Sorrow: Framing Our Attention to Suffering. Boston: Beacon Press, 1997. 113-132.

Thompson, Dawn. "Re-Inventing the world: Calculating the con/volutional integrals of holography in Nicole Brossard's Picture theory." Writing a Politics of Perception: Memory, Holography, and Women Writers in Canada. Toronto, Buffalo et London: University of Toronto Press, 2000. 16-42.

http://www.brocku.ca/cfra/voixplurielles05-02/index.html 
1 À propos des divers problèmes d'exclusion que peuvent occasionner les figures parentes de la métaphore et de I'analogie, voir Trina Grillo et Stéphanie M. Wildeman, auxquelles se réfère Knutson, ainsi que Meryl Altman et Elizabeth V. Spelman.

${ }^{2}$ Les références au roman Picture theory seront indiquées entre parenthèses avec le sigle $P T$ suivi du numéro de la page.

${ }^{3}$ Les références à l'essai «De Radical à integrals » seront indiquées entre parenthèses avec le sigle $D R$ suivi du numéro de la page. Quant aux références à d'autres essais contenus dans La Lettre aérienne, elles seront désignées par le sigle $L A$, également suivi du numéro de la page.

${ }^{4}$ Un impératif qui resurgit dans Langues obscures: « Il faudra s'entendre sur les mots 》 (52).

${ }^{5}$ Voir, par exemple, I'entrevue approfondie accordée par Nicole Brossard à l'équipe de La Nouvelle barre du jour, consacrée exclusivement à la publication de Picture theory (Cotnoir).

http://www.brocku.ca/cfra/voixplurielles05-02/index.html 